\title{
Análise da incidência e prevalência de laceração perineal de causa obstétrica em maternidade terciária de Fortaleza-CE
}

\section{Analysis of the incidence and prevalence of perineal laceration of obstetric causes in a tertiary maternity in Fortaleza-CE}

\author{
Stefanie Viana Aguiar ${ }^{1}$. Emilcy Rebouças Gonçalves². Leonardo Robson Pinheiro Sobreira Bezerra ${ }^{3}$. \\ 1 Médico, Residente de Obstetrícia e Ginecologia, Maternidade Escola Assis Chateaubriand (MEAC), Universidade Federal do \\ Ceará (UFC), Fortaleza, Ceará, Brasil. 2 Médica Obstetra e Ginecologista, Maternidade Escola Assis Chateaubriand (MEAC), \\ Fortaleza, Ceará, Brasil. 3 Doutorado em Ginecologia, Professor associado, Universidade Federal do Ceará (UFC), Coordenador \\ da Residência Médica em Obstetrícia e Ginecologia, Maternidade Escola Assis Chateaubriand (MEAC), Fortaleza, Ceará, Brasil.
}

\section{RESUMO}

As lacerações perineais obstétricas resultam em transtorno no cotidiano da mulher, seus fatores de risco incluem: uso de fórceps, trabalho de parto prolongado, nuliparidade, macrossomia fetal e episiotomia. Objetivo: avaliar a prevalência e incidência de lacerações perineais de causa obstétrica em partos vaginais. Ainda, determinar a prevalência de episiotomia e descrever fatores materno-fetais e assistenciais relacionados às lacerações perineais graves. Metodologia: estudo descritivo, documental, retrospectivo e quantitativo, realizado de abril a setembro de 2015 na Maternidade Escola Assis Chateaubriand, através de revisão de prontuário. Os dados foram analisados pelo software R (versão 3.1.2). Resultados e discussão: foram avaliados 765 partos vaginais, a prevalência das lacerações perineais foi $55 \%$, destas, $2,5 \%$ foram lacerações perineais graves $\left(3^{\circ} \mathrm{e} 4^{\circ} \mathrm{grau}\right)$. A taxa de episiotomia encontrada foi de 7,3\%. A presença de um parto vaginal prévio, ou mais, foi considerado um fator protetor para lacerações graves, $\mathrm{X}^{2}(2)=45,944^{\mathrm{a}}, \mathrm{p}=, 0000$. Gestações com 37 ou mais semanas foram avaliadas como fatores de risco para lacerações graves, $X^{2}(4)=27,637^{a}, p=0,000$. Conclusão: neste estudo, o número de lacerações perineais graves $(2,5 \%)$ e a episiotomia $(7,3 \%)$ estão compatíveis com a literatura mundial e seus fatores de risco.

Palavras-chave: Parto normal. Episiotomia. Períneo.

\section{ABSTRACT}

The obstetric perineal lacerations result in disorder in the woman's daily life, their risk factors include: use of forceps, prolonged labor, nulliparity, fetal macrosomia and episiotomy. Objective: to evaluate the prevalence and incidence of perineal lacerations of obstetric causes in vaginal deliveries. Also, determine the prevalence of episiotomy and describe maternal-fetal and asymptomatic factors related to severe perineal lacerations. Methodology: a descriptive, retrospective study with a quantitative approach. The study was performed between April and September, 2015, at Maternity School Assis Chateaubriand, through a review of medical records. Data were analyzed by software R (version 3.1.2). Results and discussion: 765 vaginal deliveries were evaluated, the prevalence of perineal lacerations was $55 \%$, of these, $2.5 \%$ were severe perineal lacerations (3rd and 4th degree). The rate of episiotomy was $7.3 \%$. The presence of a previous vaginal delivery, or more, was considered a protective factor for severe lacerations, $\mathrm{X}^{2}(2)=45.944^{\mathrm{a}}, \mathrm{p}=.0000$. Gestations with 37 or more weeks were evaluated as risk factors for severe lacerations, $\mathrm{X}^{2}(4)=27.637^{\mathrm{a}}, \mathrm{p}=0.000$. Conclusion: In this study, the number of severe perineal lacerations $(2.5 \%)$ and episiotomy $(7.3 \%)$ are compatible with the world literature and its risk factors.

Keywords: Natural childbirth. Episiotomy. Perineum.

Autor correspondente: Stefanie Viana Aguiar, Rua Oscar Bezerra, número 44, Damas, Fortaleza, Ceará. CEP: 60425-720.Telefone: +55 85 99612-8236. E-mail: stefanie_va@hotmail.com

Conflito de interesses: Não há qualquer conflito de interesses por parte de qualquer um dos autores.

Recebido em: 26 Dez 2017; Revisado em: 13 Abr 2018; Aceito em: 11 Ago 2018. 


\section{INTRODUÇÃO}

O períneo feminino tem o formato de losango e é composto por músculos, ligamentos, mucosas e estruturas ósseas. Durante o trabalho de parto essas estruturas podem sofrer lacerações espontâneas de graus variados ou instrumentalizadas (episiotomia), com consequências biológicas importantes a longo prazo como dor pélvica, prolapso genital, incontinência urinária e fecal, e dispareunia. ${ }^{1,2}$

As lacerações de trajeto de parto podem ser subdivididas em quatro tipos: primeiro grau, quando a lesão acomete apenas pele e/ou mucosa vaginal; segundo grau, quando a lesão atinge os músculos do períneo, porém, sem cometer esfíncter anal; terceiro grau, quando há prejuízo do complexo esfincteriano, podendo se subdividir em $3 \mathrm{a}$ (menos de $50 \%$ da espessura do esfíncter anal externo), 3b (mais de $50 \%$ da espessura do esfíncter anal externo) e $3 c$ (esfíncteres externo e interno são lesados); e quarto grau, quando há lesão do complexo esfincteriano externo e interno e lesão de mucosa retal. ${ }^{3}$

Um estudo com amostra de 19.442 mulheres que tiveram partos vaginais, apresentou uma frequência de $0,5 \%$ de lacerações graves (terceiro e quarto graus). ${ }^{4}$ Outro estudo realizado em Taiwan com 1.879 mulheres submetidas a partos vaginais assistidos com episiotomia mediana apresentou a prevalência de 10,9\% de lacerações graves. ${ }^{5}$

Um estudo de coorte brasileiro ${ }^{6}$ encontrou uma prevalência de $36,42 \%$ de lacerações de primeiro e segundo grau e de $0,9 \%$ de lacerações de terceiro e quarto graus. Outro estudo, também brasileiro, encontrou uma prevalência de $75,7 \%$ e de $2,5 \%$, respectivamente. ${ }^{7}$

A ocorrência de lacerações perineais durante o parto vaginal tem vários fatores potenciais, como: idade materna, paridade, uso de indutores de trabalho e analgesia, participação em aulas pré-natais, apresentação fetal, peso neonatal ao nascer e duração do segundo estágio do trabalho de parto. ${ }^{8}$

A episiotomia, tema controverso quando se trata de lacerações perineais, é uma das intervenções obstétricas mais comuns no parto. Segundo as Diretrizes para Assistência ao Parto da Organização Mundial da Saúde (OMS), ${ }^{9}$ além de não haver evidências confiáveis de que seu uso liberal ou rotineiro tenha um efeito benéfico, existem indícios claros de que pode haver dano à paciente. Nesse mesmo texto, recomenda-se o uso limitado dessa intervenção, que não deve ultrapassar a taxa de $10 \%$.

No Brasil, os índices de episiotomia ainda se mantém elevados - em muitos casos, por motivo cultural. Por muito tempo, houve a orientação de se fazer episiotomia em todas as primíparas e em multíparas submetidas ao processo em parto anterior. Estudo realizado com o objetivo de analisar a ocorrência de lesão perineal severa em parto vaginal, mostrou uma prevalência de episiotomia em $45,7 \%$ das pacientes, mas a literatura mostra que pode chegar a $90,24 \% .{ }^{10}$

Devido às sequelas no corpo feminino causada pelas lacerações perineais graves, se torna importante a avaliação de fatores assistenciais associados ao parto, com objetivo de combater os fatores evitáveis. No intuito de avaliar esses fatores, realizamos um estudo na Maternidade Escola Assis Chateaubriand (MEAC), que é um serviço de referência em atenção terciária em obstetrícia no estado do Ceará, onde ocorre um grande número de partos (em torno de 130 partos vaginais no mês). Com isso, podem ser avaliados os diferentes fatores relacionados ao trauma perineal de causa obstétrica.

\section{OBJETIVOS}

Avaliar a prevalência e incidência de lacerações perineais de causa obstétrica em partos vaginais.

Determinar a prevalência de episiotomia e descrever fatores materno-fetais e assistenciais relacionados às lacerações perineais graves.

\section{METODOLOGIA}

Este é um estudo descritivo, documental, com abordagem quantitativa, para investigar a prevalência e a incidência de lesões perineais em partos vaginais assistidos na Maternidade Escola Assis Chateaubriand (MEAC), no período de abril a setembro de 2015. A amostragem para alcançar o grau de confiança em 95\% e o erro amostral em 3,6 é de 741 mulheres, no universo de 3 milhões que representa o total de partos no Brasil em 2015.

Os critérios de inclusão são todos os partos ocorridos na MEAC no período de abril a setembro de 2015. Foram excluídas do estudo mulheres que pariram fetos malformados ou natimortos, peso do recém-nascido menor que 500 gramas, e partos vaginais que não ocorreram na MEAC.

A coleta de dados ocorreu a partir da pesquisa em prontuários. Os dados apurados preencheram um instrumento de coleta de dados, confeccionado a partir das informações contidas na ficha de monitoramento da assistência prestada ao parto e nascimento, do Ministério da Saúde, e acrescido de informações contidas no partograma, a fim de contemplar todos os possíveis fatores de risco para laceração perineal abrangidos na literatura.

A análise dos dados ocorreu através da estatística descritiva e analítica, por meio de tabulação pelo programa Microsoft Excel e pelo teste qui-quadrado $(p<0,05)$ calculado pelo software R (versão 3.1.2).

A pesquisa obedece a Resolução $N^{\circ} 466$ de 12 de dezembro de 2012, sobre pesquisa envolvendo seres humanos do Ministério da Saúde, e foi submetida à avaliação do Comitê de Ética em Pesquisa local (1.311.781).

\section{RESULTADOS}

Este estudo coletou 765 partos vaginais em uma revisão de prontuário do período de abril a setembro de 2015. A idade materna variou entre 12 e 48 anos, com média de 23,3 anos e desvio padrão de 6,7 anos. 
Nas pacientes incluídas no estudo, o número de partos vaginais prévios variou entre 0 a 8, sendo 459 (60\%) delas nulíparas. O número de cesáreas prévias variou entre 0 a 3 com maior prevalência de zero cesáreas, $729(95,2 \%)$. O número de abortos prévios foram entre 0 a 7, com 517 (67,5\%) sem abortos (Quadro 1).

Quadro 1. Distribuição do número e tipo de partos prévios, e número de abortos prévios das pacientes internadas na MEAC, no período de abril a setembro de 2015 .

\begin{tabular}{|l|l|}
\hline Número de partos vaginais prévios & Quantidades \\
\hline 0 & 459 \\
\hline 1 & 182 \\
\hline 2 & 73 \\
\hline 3 & 25 \\
\hline 4 & 12 \\
\hline 5 & 5 \\
\hline 6 & 5 \\
\hline 7 & 1 \\
\hline 8 & 3 \\
\hline Número de partos cesáreos prévios & Quantidades \\
\hline 0 & 729 \\
\hline 1 & 31 \\
\hline 2 & 2 \\
\hline 3 ou mais & 1 \\
\hline Abortos prévios & Quantidades \\
\hline 0 & 517 \\
\hline 1 & 80 \\
\hline 2 & 20 \\
\hline 3 ou mais & 5 \\
\hline & \\
\hline & \\
\hline
\end{tabular}

As gestações com fetos únicos representaram 99,5\% e 0,5\% dos gemelares dos partos vaginais; não tivemos partos vaginais de trigemelares ou mais. Dos recém-nascidos, 616 $(80,5 \%)$ foram a termo, $115(15 \%)$ com idade gestacional entre 32 semanas até 36 semanas e 6 dias, e 31 (4\%) abaixo de 31 semanas e 6 dias.

Setecentos e dezesseis pacientes entraram em trabalho de parto com a apresentação fetal cefálica, 7 com apresentação pélvica e 42 com registros ignorados.

Foram 695 inícios de trabalho de parto espontâneos. As induções foram feitas com misoprostol (38), ocitocina (14) ou com os dois (10). Das pacientes, 91,2\% foram admitidas com contrações. A qualidade das contrações durante o trabalho de parto foram divididas entre eussistolia $(50,5 \%)$, bradissistolia $(9,8 \%)$, taquissistolia $(1,5 \%)$ e ignorado $(38,2 \%)$.
No momento da admissão na MEAC, 61,5\% das pacientes apresentavam as membranas ovulares íntegras, 32,2\% com rotura das membranas ovulares, 6,3\% ignorados, e dilatação variando entre 0 a $10 \mathrm{~cm}$, sendo a maior incidência com dilatação entre 4-6 cm, o que contabilizou um total de 325 pacientes, representando $42,4 \%$ das internações. 373 (48,7\%) das pacientes foram internadas com $6 \mathrm{~cm}$ ou mais de dilatação do colo uterino.

A amniotomia foi realizada em $15,4 \%$ das pacientes, e o momento da amniotomia variou entre 6 a $10 \mathrm{~cm}$.

O uso de ocitocina foi feito em $95,2 \%$ das pacientes durante o curso do trabalho de parto ativo, no primeiro, segundo e/ou terceiro estágio. A prescrição deste hormônio ocorreu em 1 ou mais estágios num mesmo trabalho de parto. No primeiro estágio foram $89(11,6 \%)$ partos que fizeram uso da ocitocina, 91 (11,8\%) no segundo e 693 (90,5\%) no terceiro estágio.

A analgesia farmacológica foi feita em 3,8\% das pacientes, sendo 18 peridurais, 7 raquianestesia e 2 raquianestesia/ peridural. $9(1,1 \%)$ partos foram instrumentalizados com uso do fórceps. A episiotomia foi realizada em $56(7,3 \%)$ pacientes, não houve registro de episiotomia mediana e 49 foram submetidos a episiotomia médio-lateral.

As lacerações perineais ocorreram em 55\% das pacientes, sendo $27,3 \%$ (209) de $1^{\circ}$ grau, 25,2\% (193) de $2^{\circ}$ grau, $2,5 \%$ (19) de $3^{\circ}$ e $0,2 \%$ (2) de $4^{\circ}$ grau, conforme ilustrado no Gráfico 1 .

Gráfico 1. Distribuição do número de mulheres segundo o grau de laceração perineal na MEAC, no período de abril a setembro, 2015. MEAC.

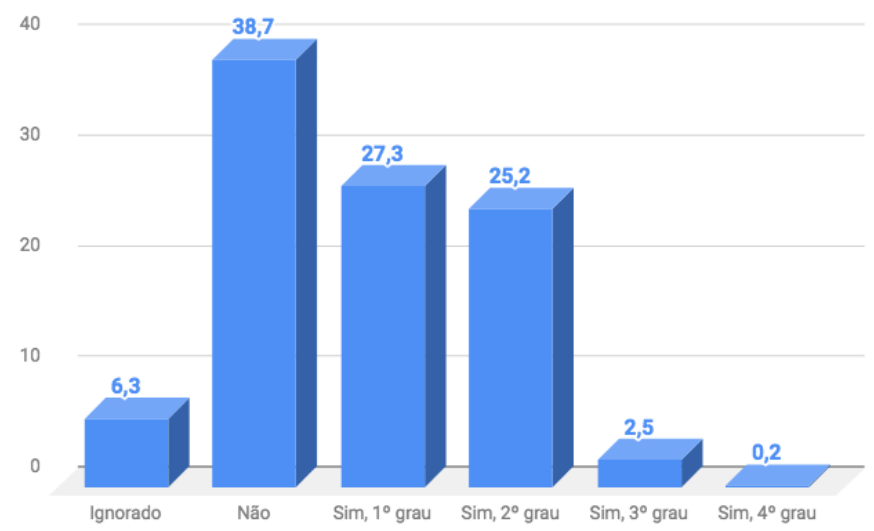

O tempo de trabalho de parto variou entre 0 a 29 horas, sendo 622 pacientes com menos de 15 horas, e 130 tiveram 1 hora ou menos de trabalho de parto. O período expulsivo variou de 0 a 330 minutos, e 11 partos tiveram mais de 120 minutos de período expulsivo.

O peso dos recém-nascidos variou de 645 gramas a 4910 gramas, $34(4,4 \%)$ tiveram o peso ao nascer maior ou igual a 4 quilos. 
Dos partos, 722 (94,3\%) ocorreram nos quartos PPP (pré-parto, parto e puerpério imediato) ou sala de parto, $20(2,6 \%)$ na emergência, $10(1,3 \%)$ no centro cirúrgico e $1(0,1 \%)$ na enfermaria. Os partos ocorridos na emergência são, na grande maioria, de pacientes admitidas em período expulsivo. Dos 10 realizados no centro cirúrgico, 7 (70\%) foram partos instrumentalizados com fórceps e episiotomia.

A assistência do parto foi feita pelos seguintes profissionais: $329(43 \%)$ com obstetra, $263(34,3 \%)$ com enfermeira/ residente de enfermagem obstétrica e 166 (21,6\%) com médico residente em obstetrícia, podendo ter ou não o auxílio de estudantes de medicina (internos).

\section{DISCUSSÃO}

O parto vaginal é uma das causas de lacerações perineais graves, que são importantes fatores de risco para disfunções do assoalho pélvico.

Lesões graves (terceiro e quarto grau) são aquelas que atingem o esfíncter anal, podendo causar problemas como fístulas ou incontinência fecal. Na Maternidade Escola Assis Chateaubriand, encontramos uma prevalência de lacerações perineais em $55 \%$ das pacientes submetidas a parto vaginal. Destas, 52,5\% foram leve (primeiro e segundo grau) e 2,5\% foram graves (terceiro e quarto grau).

Os fatores de risco descritos para lesões de terceiro e quarto grau são: parto instrumental, nuliparidade, segundo estágio do trabalho de parto prolongado, grande peso ao nascer, distócia de ombros, variedade fetal e episiotomia. ${ }^{8,11}$

Lesões perineais de terceiro e quarto graus são mais frequentes em nulíparas e em casos em que há uso de ocitocina durante o trabalho de parto. Nesse mesmo estudo, o principal fator de risco identificado para lacerações graves foi o uso de parto instrumentalizado. ${ }^{4}$

O estudo avaliou retrospectivamente 765 partos vaginais ocorridos na MEAC e descreveu os principais fatores de risco para lacerações graves. Observamos algumas variáveis como número de partos cesarianas prévios, abortamentos prévios, dilatação no momento em que foi feito a amniotomia, dilatação do colo de útero no momento da admissão, tempo de trabalho de parto, tempo de período expulsivo e idade da paciente. Todas mantiveram ocorrências proporcionais entre as pacientes sem lacerações, com lacerações leves $\left(1^{\circ}\right.$ e $2^{\circ}$ grau) e lacerações graves $\left(3^{\circ}\right.$ e $4^{\circ}$ grau $)$.

$\mathrm{Na}$ avaliação estatística, notamos que a presença de um parto vaginal prévio, ou mais, é considerado um fator protetor para lacerações graves, $X^{2}(2)=45,944^{\mathrm{a}}, \mathrm{p}=, 0000$. Em relação ao peso fetal, observamos que recém-nascidos com peso $>4000 \mathrm{~g}$ não tiveram associação com as lacerações, $X^{2}(2)=0,097^{\mathrm{a}}$, $p=0,953$, ns. Nas outras variáveis, não conseguimos fazer sua avaliação estatística devido ao seu baixo registro ou sua qualidade para o estudo.

Não encontramos significância estatística no uso de analgesia farmacológica com as lacerações graves, $X^{2}(4)=4,840^{\mathrm{a}}, p=$ 0,304 .

As variáveis apresentação do primeiro feto, presença das membranas amnióticas íntegras na admissão, utilização do partograma, tipo de parto e tipo de gestação, mostraram porcentagens semelhantes entres as pacientes que tiveram ou não lacerações.

Gestações com 37 ou mais semanas foram avaliadas como fatores de risco para lacerações graves, $X^{2}(4)=27,637^{\mathrm{a}}, p=0,000$.

Partos ocorridos na emergência foram fatores de proteção para as lacerações graves, $X^{2}(10)=21,526^{\mathrm{a}}, p=0,018$. Após analisar essa variável, notamos a presença de 13 pacientes com período expulsivo e 17 delas com pelo menos um parto vaginal prévio, o que provavelmente colaborou para o favorecimento ou viés dessa associação.

O parto induzido (com misoprostol ou ocitocina) teve uma tendência como fator de risco, $X^{2}(2)=5,956^{\mathrm{a}}, p=0,051$. O uso de ocitocina no primeiro e segundo estágios ficou com avaliação estatística comprometida devido à quantidade e qualidade dos dados colhidos. O uso de ocitocina no terceiro estágio é um procedimento rotineiro do hospital.

No Quadro 2 apresentamos os principais indicadores avaliados no estudo e sua significância estatística.

Quadro 2. Distribuição das características clínicas e obstétricas das pacientes internadas na MEAC para partos normais, no período de abril a setembro de 2015 .

\begin{tabular}{|l|l|l|l|l|}
\hline & \multicolumn{4}{|c|}{ Lacerações } \\
\hline & Não & $\mathbf{1}^{\mathbf{0}}$ e 2 \\
\hline
\end{tabular}




\section{CONCLUSÃO}

No presente estudo, conclui-se que os fatores protetores para lacerações graves incluem a presença de pelo menos um parto vaginal prévio.

Como fatores de risco, tivemos as gestações com 37 ou mais semanas. Nos partos induzidos, notamos uma tendência, como

\section{REFERÊNCIAS}

1. Goh R, Goh D, Ellepola H. Perineal tears - a review. Aust J Gen Pract. 2018;47(1/2):35-8.

2. Dunn AB, Paul S, Ware LZ, Corwin EJ. Perineal injury during chilbirth increases risk of postpartum depressive symptoms and inflammatory markers. J Midwifery Womens Health. 2015;60(4):42836.

3. Royal College of Obstetricians and Gynaecologists. The management of third- and fourth-degree perineal tears [Internet]. 2015 [acesso em: 01 jun 2015]. Disponível em: https://www.rcog. org.uk/globalassets/documents/guidelines/gtg-29.pdf

4. Schmitz T, Alberti C, Andriss B, Moutafoff C, Oury JF, Sibony O. Identification of women at high risk for severe perineal lacerations. Eur J Obstet Gynecol Reprod Biol. 2014;182:11-5.

5. Hsieh WC, Liang CC, Wu D, Chang SD, Chueh HY, Chao AS. Prevalence and contributing factors of severe perineal damage following episiotomy-assisted vaginal delivery. Taiwan J Obstet Gynecol. 2014;53(4):481-5. fator de risco, para as lacerações graves.

Neste estudo, o número de lacerações perineais graves (2,5\%) e a episiotomia $(7,3 \%)$ estão compatíveis com a literatura mundial e seus fatores de risco.

6. Oliveira LS, Brito LG, Quintana SM, Duarte G, Marcolin AC. Perineal trauma after vaginal delivery in healthy pregnant women. Sao Paulo Med J. 2014;132(4):231-8.

7. Monteiro MV, Pereira GM, Aguair RA, Azevedo RL, CorreiaJunior MD, Reis ZS. Risk factors for severe obstetric perineal lacerations. Int Urogynecol J. 2016; 27(1):61-7.

8. Laganà AS, Terzic M, Dotlic J, Sturlese E, Palmara V, Retto G, et al. The role of episiotomy in prevention of genital lacerations durang vaginal deliveries - resuls from two european centers. Ginekol Pol. 2015;86(3):168-75.

9. Organização Mundial de Saúde. Maternidade segura: assistência ao parto normal: um guia prático: relatório de um grupo técnico. Brasília: Ministério da Saúde; 1996.

10. Santos JO, Bolanho IC, Mota JQ, Coleoni L, Oliveira MA. Frequência de lesões perineais ocorridas nos partos vaginais em uma instituição hospitalar. Esc Anna Nery. 2008;12(4):658-63.

11. Friedman AM, Ananth CV, Prendergast E, D'Alton ME, Wright JD. Evaluation of third-degree and fourth-degree laceration rates as quality indicators. Obstet Gynecol. 2015;125(4):927-37.

\section{Como citar:}

Aguiar SV, Gonçalves ER, Bezerra LR. Análise da incidência e prevalência de laceração perineal de causa obstétrica em maternidade terciária de Fortaleza-CE. Rev Med UFC. 2019 jan-mar;59(1):39-43. 